

\section{William G. Christen,}

Department of Medicine, Brigham and Women's Hospital, Boston, MA, USA

John Fingert,

Department of Ophthalmology, College of Medicine, University of lowa, lowa City, IO, USA

Department of A natomy/Cell Biology, College of Medicine, University of lowa, lowa City, IO, USA

Douglas Gaasterland,

Eye Doctors of Washington, Chevy Chase, MD, USA

Terry Gaasterland,

Scripps Genome Center, University of California at San Diego, San Diego, CA, USA

Peter Kraft,

Department of Biostatistics, Harvard School of Public Health, Boston, MA, USA

Richard K. Lee,

Bascom Palmer Eye Institute, University of Miami Miller School of Medicine, Miami, FL, USA

Paul R. Lichter,

Department of Ophthalmology and V isual Sciences, University of Michigan, Ann Arbor, MI, USA

Yutao Liu,

Department of Ophthalmology, Duke University Medical Center, Durham, NC, USA

Department of Medicine, Duke University Medical Center, Durham, NC, USA

Catherine A. McCarty,

Essentia Institute of Rural Health, Duluth, MN, USA

\section{Sayoko E. Moroi,}

Department of Ophthalmology and V isual Sciences, University of Michigan, Ann Arbor, MI, USA

Julia E. Richards,

Department of Ophthalmology and V isual Sciences, University of Michigan, Ann Arbor, MI, USA

Tony Realini,

Department of Ophthalmology, WVU Eye Institute, Morgantown, WV, USA

Joel S. Schuman,

Department of Ophthalmology, UPMC Eye Center, University of Pittsburgh, Pittsburgh, PA, USA

William K. Scott,

Institute for Human Genomics, University of Miami Miller School of Medicine, Miami, FL, USA

Kuldev Singh,

Department of Ophthalmology, Stanford University, Palo Alto, CA, USA

Arthur J. Sit,

Department of Ophthalmology, Mayo Clinic, Rochester, MN, USA

Douglas Vollrath,

Department of Genetics, Stanford University, Palo Alto, CA, USA

Gadi Wollstein, 
Department of Ophthalmology, UPMC Eye Center, University of Pittsburgh, Pittsburgh, PA, USA

Donald J. Zack,

Wilmer Eye Institute, Johns Hopkins University Hospital, Baltimore, MD, USA

Kang Zhang,

Department of Ophthalmology, Hamilton Eye Center, University of California, San Diego, CA, USA

Margaret A. Pericak-Vance,

Institute for Human Genomics, University of Miami Miller School of Medicine, Miami, FL, USA

R. Rand Allingham,

Department of Ophthalmology, Duke University Medical Center, Durham, NC, USA

Robert N. Weinreb,

Department of Ophthalmology, Hamilton Eye Center, University of California, San Diego, CA, USA

Jonathan L. Haines, and

Center for Human Genetics Research, Vanderbilt University, Nashville, TN, USA

Janey L. Wiggs

Department of Ophthalmology, Harvard Medical School, Massachusetts Eye and Ear Infirmary, Boston, MA, USA

Janey L. Wiggs: janey_wiggs@meei.harvard.edu

\section{Abstract}

Primary open-angle glaucoma (POAG) is a leading cause of blindness worldwide. Using genomewide association single-nucleotide polymorphism data from the Glaucoma Genes and Environment study and National Eye Institute Glaucoma Human Genetics Collaboration comprising 3,108 cases and 3,430 controls, we assessed biologic pathways as annotated in the KEGG database for association with risk of POAG. After correction for genic overlap among pathways, we found 4 pathways, butanoate metabolism (hsa00650), hematopoietic cell lineage (hsa04640), lysine degradation (hsa00310) and basal transcription factors (hsa03022) related to POAG with permuted $p<0.001$. In addition, the human leukocyte antigen (HLA) gene family was significantly associated with POAG $(p<0.001)$. In the POAG subset with normal-pressure glaucoma (NPG), the butanoate metabolism pathway was also significantly associated $(p<0.001)$ as well as the MAPK and Hedgehog signaling pathways (hsa04010 and hsa04340), glycosaminoglycan biosynthesis-heparan sulfate pathway (hsa00534) and the phenylalanine, tyrosine and tryptophan biosynthesis pathway (hsa0400). The butanoate metabolism pathway overall, and specifically the aspects of the pathway that contribute to GABA and acetyl-CoA metabolism, was the only pathway significantly associated with both POAG and NPG.

Collectively these results implicate GABA and acetyl-CoA metabolism in glaucoma pathogenesis, and suggest new potential therapeutic targets. 


\section{Introduction}

Glaucoma is the second leading cause of blindness worldwide, with an estimated 60 million people affected (Quigley 2011; Quigley and Broman 2006). In the United States, primary open-angle glaucoma (POAG) is the most common type of glaucoma (Friedman et al. 2004). POAG is an age-related progressive optic nerve degeneration caused by irreversible destruction of retinal ganglion cells eventually leading to blindness. Risk factors for POAG, aside from age, include elevated intraocular pressure, family history and African-American race (Caprioli and Varma 2011; Racette et al. 2003; Shin et al. 1977). A subgroup of POAG patients develops optic nerve degeneration without elevation of intraocular pressure (IOP) and this POAG subset is called normal-pressure glaucoma (NPG).

POAG is genetically and phenotypically complex (Fan and Wiggs 2010). Recent genomewide association studies (GWAS) of large case-control and population-based cohorts have identified genes and genomic regions associated with POAG risk including an intergenic region between caveolins 1 and 2 (CAV1/CAV2) (Thorleifsson et al. 2010; Wiggs et al. 2011), TMCO1 and CDKN2BAS (Burdon et al. 2011; Wiggs et al. 2012), SIX1/SIX6 (Wiggs et al. 2012) and 8q22 in the NPG subgroup (Wiggs et al. 2012).

While these GWAS have identified multiple genes potentially involved in glaucoma pathogenesis, the stringent correction for multiple testing and type I error identifies only variants with the largest main effects as statistically significant (Manolio et al. 2008). Many biologically meaningful associations of smaller effect size may go undetected, including variants with smaller effect size that in aggregate could reveal novel biological pathways or systems underlying disease susceptibility. Pathway-based analysis groups gene variants into biologically meaningful entities to distinguish the actual from the false positive associations. In short, this type of analysis can be used to uncover additional genotype-phenotype associations that the single-allele GWAS analysis may have missed.

Pathway analysis by randomization incorporating structure (PARIS) is a pathway analysis algorithm that uses single-allele GWAS data and assigns significance to a pathway through permutation of the genome rather than permutation of affection status (Yaspan et al. 2011). Through genomic randomization, PARIS accounts for multiple testing, gene size/SNP coverage and linkage disequilibrium (LD) biases present in the original GWAS dataset. Here, we report the use of PARIS in a hypothesis-independent approach to identify novel pathways and genes associated with POAG and the NPG subgroup with optic nerve degeneration independent of IOP.

\section{Results}

The demographics and ocular characteristics for GLAUGEN and NEIGHBOR study participants have been reported previously (Wiggs et al. 2011, 2012, 2013). We used the GWAS $p$ values from the case-control meta-analysis in the NEIGHBOR and GLAUGEN datasets and executed the PARIS algorithm (Yaspan et al. 2011) using the KEGG pathways $(n=209)$ as a framework. We found significant association with POAG in 14 of the 209 pathways in the KEGG database (Table 1). These pathways were loosely grouped into three 
KEGG categories: metabolism, cellular adhesion and signaling, and autoimmune disorders. For a complete list of all 209 pathways and their permuted $p$ values, see Supplemental Table 1.

We next investigated possible relationships among the 14 pathways associated with POAG. We found that nine of these pathways contain a substantial amount of genic overlap due to the HLA gene family (HLA-A, HLA-B, HLA-C, HLA-E, HLA-F, HLA-G, HLA-DMA, HLADMB, HLADOA, HLA-DOB, HLA-DPA1, HLA-DPB1, HLA-DQA1, HLA-DQA2, HLA$D Q B 1, H L A-D R A, H L A-D R B 1, H L A D R B 5, H L A-E, H L A-F$, and HLA-G). All nine of the pathways did not have all of these genes, but most of these pathways have several and all nine pathways include $H L A-D O B, H L A-D Q A 2$ and $H L A-C$. A gene-based permutation test using PARIS (each gene within the pathway is considered as a separate entity), identified significant association $(p<0.001)$ for these three HLA gene family members (Fig. 1). To test the impact of all 21 HLA genes, we removed them from the nine pathways containing HLA gene family members and re-ran PARIS and found that all nine of these pathways were no longer statistically significant. Although the overall pathways were not significant, several genes within the 9 HLA-containing pathways continued to demonstrate interesting association using the gene-based test (Fig. 1). The viral myocarditis pathway (hsa05416) contains CAVI (Caveolin 1) previously shown to be associated with POAG (Thorleifsson et al. 2010; Wiggs et al. 2011). In the cell adhesion pathway, JAM2 is a component of the neural retina and retinal pigment epithelium (Daniele et al. 2007) and PVRL3 has previously been shown to contribute to ocular developmental defects (Lachke et al. 2012).

Next we used the gene-based permutation test to identify the genes within each of the remaining five pathways with significant association $(p<0.001)$. Three of the remaining five pathways - butanoate metabolism, lysine degradation, and limonene and pinene degradation — contained the significantly associated genes $A L D H 2$ and ALDH3A2 (Fig. 1). Two other genes in the lysine degradation pathway are also associated with POAG (GLT25D2, EHMTA) and one other gene in the butanoate metabolism pathway $(B D H 1)$ was significantly associated with POAG. The only significant genes in the limonene and pinene degradation pathway are $A L D H 2$ and $A L D H 3 A 2$. Two genes in the Leishmaniasis pathway overlap with the hematopoietic cell lineage pathway. The significantly associated genes in the basal transcription factor pathway were independent of all other pathways (Fig. 1). Overall, after correcting for genic overlap, we identified four pathways (butanoate metabolism, lysine degradation, hematopoietic cell lineage and basal transcription factor) and HLA genes $H L A-D O B, H L A-D Q A 2$ and $H L A-C$ associated with POAG.

In the NEIGHBOR and GLAUGEN single-allele meta-analysis, we discovered that the NPG subgroup of cases was driving the genome-wide signals despite a sample size $(n=720)$ of less than half of the high-pressure glaucoma (HPG) subset ( $n=1,669)$ (Wiggs et al. 2012). As POAG is phenotypically heterogeneous we used PARIS to test the 209 KEGG pathways in the NPG subset (Supplemental Table 1). Seven pathways were statistically significant in the NPG subgroup (Fig. 2; Table 2). Using the gene-based permutation test to identify the genes with significant association $(p<0.001)$ with NPG we found that there was substantial genic overlap between the MAPK signaling pathway and the Fc Epsilon RI signaling pathway, and between the butanoate pathway and the synthesis and degradation of ketone 
bodies pathway, leaving five pathways significantly associated with NPG: the MAPK signaling pathway, butanoate pathway, Hedgehog signaling, phenylalanine, tyrosine and tryptophan biosynthesis, and the glycosaminoglycan- heparan sulfate biosynthesis pathway. Of these the butanoate metabolism pathway is the only pathway that was significant in both the overall POAG analysis and the NPG analysis.

While the entire butanoate metabolism pathway may affect POAG/NPG etiology, not all of the genes within it may be of interest. Using the gene-based permutation test we evaluated each gene within the pathway to identify those genes important to the signal of the pathway as a whole for both POAG overall and for the NPG subgroup. Of the 35 genes in the butanoate pathway, 9 were nominally significant for POAG overall and 8 were nominally significant in the NPG subgroup (Table 3). Among these 17 are three genes encoding enzymes that regulate GABA synthesis (GAD1, ABAT, ALDH5A1), an important inhibitory neurotransmitter in the mammalian central nervous system and retina (Bringmann et al. 2013). Two genes had permutated $p$ values of $<0.001$ for both POAG and NPG: ALDH3A2, an aldehyde dehydrogenase involved in Sjogren Larsson syndrome, a neurodevelopmental disorder that includes ocular phenotypes (van der Veen et al. 2010) and BDH1, (3hydroxybutyrate dehydrogenase), a mitochondrial protein that oxidizes 3-hydroxybutyrate to form acetoacetate and NA DH+ (Maurer et al. 2011). Of the 8 genes with at least nominal association with NPG, 7 of them influence acetyl-CoA production in mitochondria (Fig. 3). Using an alternative and different analytic algorithm for gene-based analysis (VEGAS) that sums the Chi square statistic for all variants assigned to each gene and accounts for LD using a Monte Carlo simulation (Liu et al. 2010), we also found at least nominal evidence of association for $A L D H 1 B 1$ and $B D H 1$ with POAG and ALDH3A2, ALDH5A1, BDH1 and $E C H S 1$ with NPG providing additional support for these results (Table 3).

\section{Discussion}

In this study, we performed a hypothesis-independent pathway analysis using the KEGG pathways and GWAS data from a large POAG/NPG dataset (Wiggs et al. 2012). Pathway analyses make it possible to use biologically meaningful entities to identify variants that individually may not reach genome-wide statistical significance, but in aggregate can be statistically associated with a disease trait. We found that the butanoate metabolism pathway (hsa00650) is significantly associated with both POAG overall and the NPG subgroup, a result that includes significant association of genes contributing to the formation of 4aminobutanoate (GABA) (Fig. 3). GABA is a neurotransmitter that has a critical role in visual responsiveness (Zhang and McCall 2012). GABA activity has been implicated in retinal ganglion cell degeneration in a rat hypertensive glaucoma model, and in a mouse model of glaucoma (Moreno et al. 2008; Okumichi et al. 2008). In addition, glutamate, a precursor to GABA, is excitotoxic in retinal ganglion cells (Nguyen et al. 2011). Interestingly, visual field loss is a side effect of the antileptic, Vigabatrin, an inhibitor of GABA transaminase (encoded by ABAT) (Lawden et al. 1999). Vigabatrin-related visual field loss resembles glaucomatous loss and is likely due to biochemical disruption of the photoreceptor-Mueller cell-retinal ganglion cell complex (Clayton et al. 2012). In one study $44 \%$ of cases (those with seizures taking vigabatrin) had evidence of visual field loss compared to $7 \%$ of controls (Lux et al. 2004). 
In addition to GABA metabolism, the butanoate pathway includes enzymes responsible for acetyl-CoA biosynthesis and required for AT P production in mitochondria. Interestingly, of the 8 butanoate pathway genes associated with NPG, 7 of them influence acetyl-CoA production (Fig. 3). Retinal ganglion cells have high-energy requirements and are dependent on mitochondrial AT P production for survival (Yu et al. 2013), and previous studies have suggested that mitochondrial dysfunction is a risk factor for ganglion cell death in glaucoma (Chrysostomou et al. 2013; Lee et al. 2012). Our results support these hypotheses and suggest that further research on mitochondrial genes, proteins and function in glaucoma would be of interest.

The butanoate pathway also impacts thiamine synthesis, and thiamine deficiency is known to cause optic atrophy and other neurologic problems (Sedel et al. 2008). Pyruvate dehydrogenase (lipoamide) beta $(P B H B)$, responsible for converting pyruvate to a thiamine precursor, was significantly associated $(p<0.001)$ with NPG providing support for a role of thiamine biosynthesis in optic nerve degeneration in glaucoma. Vitamin B1 (Thiamine) intake has also been shown to be associated with a reduced risk of POAG (Ramdas et al. 2012).

We also found a significant association with the HLA family of genes potentially indicating a contribution of immune responsiveness to glaucoma pathogenesis. A number of studies have suggested a role for the immune system in glaucoma (Wax 2011); in particular, genetic associations between POAG and HLA DRB1 and DQB1 have been evaluated in various populations, although with inconsistent results (Gil-Carrasco et al. 1999; Suzuki et al. 2010). It is possible that the differential North to South distribution of HLA alleles in Europeans may create spurious associations between HLA SNPs and POAG that are not fully corrected by including the selected eigenvectors in the logistic regression models. Nevertheless, our findings suggest that further investigation into the role of HLA genes and glaucoma may be of interest.

Two other pathways were associated with POAG overall: the Basal transcription factor pathway and the hematopoietic cell lineage pathway that includes EPO, encoding erythropoietin which has been shown to be neuroprotective in an animal model of glaucoma (Sullivan et al. 2012). While mutations in genes coding for several transcription factors cause Mendelian early-onset glaucoma (Fan and Wiggs 2010), basal transcription factors have not been associated with common complex forms of glaucoma such as POAG or NPG. The robust association of $C D K N 2 B A S$ with POAG and NPG (Burdon et al. 2011; Wiggs et al. 2012), a long noncoding RNA that regulates expression of $C D K N 2 B$ (p16) an inhibitor of cell cycle regulatory protein, CDK4 (cyclin-dependent kinase 4) (Wan et al. 2013), suggests that cell cycle progression may be an important regulator of glaucoma pathogenesis and the basal transcription factors also contribute to cell cycle regulation overall.

In addition to the butanoate pathway, three other pathways associated with NPG are of interest. The MAPK signaling pathway can affect neuronal development and degeneration (Dapper et al. 2013). Associated genes in this pathway, RAC3 (Albertinazzi et al. 2003) and PRKCA (Guo et al. 2012), influence ocular neurite outgrowth and axon guidance, respectively. The Hedgehog signaling pathway was associated with NPG overall, and 
several associated genes within this pathway have important roles in ocular development (WNT4, WNT8b, WNT6, BMP7) (Maurus et al. 2005; Fokina and Frolova 2006; Martinez et al. 2009; Wyatt et al. 2010). Finally EXT2, a member of the glycosaminoglycan biosynthesis-heparan sulfate pathway, also contributes to WNT signaling and to axon sorting in the optic tract (Lee et al. 2004).

There are several limitations to our study. First, our approach used the collection of pathways annotated in the KEGG database, which, while comprehensive, is not exhaustive and is limited to current knowledge published in the literature. Second, although we have employed a rigorous permutation test to reduce false positives, no other current POAG/NPG data set of comparable size is currently available to replicate the findings we present here. Further research will be necessary to confirm the relevance of these pathways to POAG and NPG.

In summary, we report a hypothesis-independent, pathway-based analysis from GWAS data in a large POAG/NPG study population. After correcting for genic overlap and multiple comparisons to random pathways with comparable genomic structure our approach identified 4 pathways associated with POAG and 5 associated with the NPG subgroup, highlighting the complex genetic nature of glaucoma. One of these pathways, butanoate metabolism (hsa00650), was significantly associated with both POAG and NPG. Although our findings require confirmation by functional studies or in other study populations, these results suggest that features of butanoate metabolism, specifically GABA signaling and acetyl-coA metabolism are important factors in glaucoma development and are worthy of further investigation.

\section{Methods}

\section{NEIGHBOR and GLAUGEN study populations}

The NEIGHBOR and GLAUGEN case-control datasets have been described in detail previously (Wiggs et al. 2011, 2012, 2013). Briefly, The GLAUGEN dataset consists of 976 cases and 1,140 controls drawn from the Genetic Etiologies of Primary Open-angle Glaucoma (GEP), the Nurses' Health Study (NHS) and the Health Professionals Follow-up study (HPFS). The GEP is a clinic-based case-control set, and the NHS and HPFS are casecontrol sets nested within population-based studies. The institutional review boards of the Massachusetts Eye and Ear Infirmary, Harvard School of Public Health and the Brigham and Women's Hospital approved this study. 2,132 cases and 2,290 controls for the NEIGHBOR study were collected from 12 sites. For those subject recruitments and these GWAS, approval was obtained from the institutional review boards of the University of Pittsburgh, Johns Hopkins University, Duke University (Duke clinic and CAT HGEN), University of West Virginia, University of Miami, University of Michigan (Michigan clinic and CIGTS and AGIS), Stanford University, Marshfield Clinic, and the University of California, San Diego. 


\section{Single allele and meta-analysis}

We performed logistic regression to assess the association between individual SNPs and POAG using PLINK v1.07 (Purcell et al. 2007). For GLAUGEN, the logistic regression model included age, gender, study site, DNA source, DNA extraction method and three eigenvectors (EV 1, 2 and 6). For NEIGHBOR, the logistic regression model included age, gender, study site and two eigenvectors (EV1 and 2). Principal component analyses (using EIGENSOFT http://www.hsph.harvard.edu/alkes-price/software/) for both the POAG group vs controls and the NPG subgroup vs. controls are shown in Supplemental Fig.1A and 1B. Quantile-quantile plots were used to estimate genomic inflation factors that were 1.009 for GLAUGEN and 1.034 for NEIGHBOR. Combined meta-analysis of the GLAUGEN and NEIGHBOR datasets was done using the METAL software package (Willer et al. 2010). We analyzed each study using logistic regression as described previously (Wiggs et al. 2011, 2012). Then, we combined the results using the inverse-weighted variance method based on the regression coefficients and standard errors estimated from each study as implemented in the program METAL. The GENOMIC CONTROL option was set to ON to adjust for genomic inflation differences between the studies (Wiggs et al. 2012).

\section{PARIS}

Results from the meta-analysis of the GLAUGEN and NEIGHBOR studies were used for the pathway analysis which was done using the PARIS pathway analysis software package (Yaspan et al. 2011). Biologic pathways were identified using the KEGG (Kyoto Encyclopedia of Genes and Genomes) pathway database for homo sapiens (hsa), version 54.1 (Kanehisa and Goto 2000). SNPs from the GWAS were considered to reside in a pathway gene if the SNP fell within the ENSEMBL genomic interval $\pm 50 \mathrm{~kb}$ to either side of the gene. If the overlap included another gene in the pathway, the overlapping SNP(s) were counted once. We set a threshold of a single-allele $p$ value of $<0.05$ as nominally significant for use within the PARIS analysis. PARIS utilizes a permutation test to determine the significance of its pathway analyses. We used 1,000 permutations for this analysis. A KEGG pathway was considered to be significant at the $p<0.001$ level if none of the 1,000 randomized pathways had more significant SNP signals than the actual pathway. To determine which of the genes in each pathway were contributing to the significant signal in the pathway, we used the "I" (Investigate) option within PARIS. Gene-based results were also investigated using VEGAS (Liu et al. 2010), using the web-based interface (http:// gump.qimr.edu.au/VEGAS/) and the default parameters, which includes the HapMap CEU for assessing LD.

\section{Supplementary Material}

Refer to Web version on PubMed Central for supplementary material.

\section{Acknowledgments}

The Harvard Glaucoma Center of Excellence and Margolis fund (Boston, MA) support LRP and JLW. LRP, JER and JLW are also supported by Research to Prevent Blindness, Inc. (New York, NY). The Arthur Ashley Foundation also supports Dr. Pasquale. The Glaucoma Research Foundation (San Francisco, CA), American Health Assistance Foundation (Clarksburg, MD), and the Glaucoma Foundation (New York, NY) support YL. The following National Institutes of Health Grants support the maintenance of the Nurses Health Study and Health 
Professionals Follow-up, allowing these health professionals to contribute to this analysis: CA87969, CA49449, UM1 CA167552, and HL35464. The following Grants from the National Human Genome Research Institute (Bethesda, MD) supported GLAUGEN: HG004728 (LRP), HG004424 (Broad Institute to support genotyping), HG004446 (C. Laurie, U. Washington, to support genotype data cleaning and analysis). Genotyping services for the NEIGHBOR study were provided by the Center for Inherited Disease Research (CIDR) and were supported by the National Eye Institute through Grant HG005259-01 (JLW). In addition, CIDR is funded through a federal contract from the National Institutes of Health to The Johns Hopkins University, contract number HHSN268200782096C. The National Eye Institute (Bethesda, MD) through ARRA Grants EY015872 (JLW) and EY019126 (MAH) supported the collection and processing of samples for the NEIGHBOR dataset. Funding for the collection of cases and controls was provided by National Institutes of Health (Bethesda, MD) Grants: EY015543 (RRA), EY006827 (DG), HL073389 (Hauser, E), EY13315 (MAH), EY09611 (Hankinson, S), EY015473 (LRP), EY009149 (PRL), HG004608 (CAM), EY008208 (Medeiros, P.), EY015473 (LRP), EY012118 (MAP-V), EY015682 (TR), EY011671 (JER), EY09580 (JER), EY013178 (JSS), EY015872 (JLW), EY010886 (JLW), EY009847 (JLW), EY011008 (Zangwill, L), EY144428 (KZ), EY144448 (KZ), EY18660 (KZ). None of the authors have any commercial interests in the subject of the manuscript or in entities discussed in the manuscript. BL Yaspan and JN Cooke Bailey were supported in part by NIH Grant T32EY021453.

\section{References}

Albertinazzi C, Za L, Paris S, de Curtis I. ADP-ribosylation factor 6 and a functional PIX/p95-APP1 complex are required for Rac1B-mediated neurite outgrowth. Mol Biol Cell. 2003; 14:1295-1307. [PubMed: 12686588]

Bringmann A, Grosche A, Pannicke T, Reichenbach A. GABA and glutamate uptake and metabolism in retinal glial (Müller) cells. Front Endocrinol (Lausanne). 2013; 4:48. [PubMed: 23616782]

Burdon KP, Macgregor S, Hewitt AW, Sharma S, Chidlow G, Mills RA, Danoy P, Casson R, Viswanathan AC, Liu JZ, Landers J, Henders AK, Wood J, Souzeau E, Crawford A, Leo P, Wang JJ, Rochtchina E, Nyholt DR, Martin NG, Montgomery GW, Mitchell P, Brown MA, Mackey DA, Craig JE. Genome-wide association study identifies susceptibility loci for open angle glaucoma at TMCO1 and CDKN2B-AS1. Nat Genet. 2011; 43:574-578. [PubMed: 21532571]

Caprioli J, Varma R. Intraocular pressure: modulation as treatment for glaucoma. Am J Ophthalmol. 2011; 152:340-344. [PubMed: 21855671]

Chrysostomou V, Rezania F, Trounce IA, Crowston JG. Oxidative stress and mitochondrial dysfunction in glaucoma. Curr Opin Pharmacol. 2013; 13:12-25. [PubMed: 23069478]

Clayton LM, Devile M, Punte T, Haan GJ, Sander JW, Acheson JF, Sisodiya SM. Patterns of peripapillary retinal nerve fiber layer thinning in vigabatrin-exposed individuals. Ophthalmol. 2012; 119:2152-2160.

Daniele LL, Adams RH, Durante DE, Pugh EN, Philp NJ. Novel distribution of junctional adhesion molecule-C in the neural retina and retinal pigment epithelium. J Comp Neurol. 2007; 505:166-176. [PubMed: 17853450]

Dapper JD, Crish SD, Pang IH, Calkins DJ. Proximal inhibition of p38 MAPK stress signaling prevents distal axonopathy. Neurobiol Dis. 2013; 59C:26-37. [PubMed: 23859799]

Fan BJ, Wiggs JL. Glaucoma: genes, phenotypes, and new directions for therapy. J Clin Invest. 2010; 120:3064-3072. [PubMed: 20811162]

Fokina VM, Frolova EI. Expression patterns of Wnt genes during development of an anterior part of the chicken eye. Dev Dyn. 2006; 235:496-505. [PubMed: 16258938]

Friedman DS, Wolfs RC, O'Colmain BJ, Klein BE, Taylor HR, West S, Leske MC, Mitchell P, Congdon N, Kempen J. Prevalence of open-angle glaucoma among adults in the United States. Arch Ophthalmol. 2004; 122:532-538. [PubMed: 15078671]

Gil-Carrasco F, Vargas-Alarcón G, Zúñiga J, Tinajero-Castañeda O, Hernández-Martinez B, Hernández-Pacheco G, Rodríguez-Reyna TS, Hesiquio R, Gamboa R, Granados J. HLA-DRB and HLA-DQB loci in the genetic susceptibility to develop glaucoma in Mexicans. Am J Ophthalmol. 1999; 128:297-300. [PubMed: 10511023]

Guo D, Standley C, Bellve K, Fogarty K, Bao ZZ. Protein kinase Ca and integrin-linked kinase mediate the negative axon guidance effects of Sonic hedgehog. Mol Cell Neurosci. 2012; 50:8292. [PubMed: 22521536]

Kanehisa M, Goto S. KEGG: kyoto encyclopedia of genes and genomes. Nucleic Acids Res. 2000; 28:27-30. [PubMed: 10592173] 
Lachke SA, Higgins AW, Inagaki M, Saadi I, Xi Q, Long M, Quade BJ, Talkowski ME, Gusella JF, Fujimoto A, Robinson ML, Yang Y, Duong QT, Shapira I, Motro B, Miyoshi J, Takai Y, Morton CC, Maas RL. The cell adhesion gene PVRL3 is associated with congenital ocular defects. Hum Genet. 2012; 131:235-250. [PubMed: 21769484]

Lawden MC, Eke T, Degg C, Harding GF, Wild JM. Visual field defects associated with vigabatrin therapy. J Neurol Neurosurg Psychiatry. 1999; 67:716-722. [PubMed: 10567485]

Lee JS, von der Hardt S, Rusch MA, Stringer SE, Stickney HL, Talbot WS, Geisler R, NüssleinVolhard C, Selleck SB, Chien CB, Roehl H. Axon sorting in the optic tract requires HSPG synthesis by ext2 (dackel) and extl3 (boxer). Neuron. 2004; 44:947-960. [PubMed: 15603738]

Lee S, Sheck L, Crowston JG, Van Bergen NJ, O’Neill EC, O’Hare F, Kong YX, Chrysostomou V, Vincent AL, Trounce IA. Impaired complex-I-linked respiration and AT P synthesis in primary open-angle glaucoma patient lymphoblasts. Invest Ophthalmol Vis Sci. 2012; 53:2431-2437. [PubMed: 22427588]

Liu JZ, McRae AF, Nyholt DR, Medland SE, Wray NR, Brown KM, Hayward NK, Montgomery GW, Visscher PM, Martin NG, Macgregor S. Investigators AMFS. A versatile gene-based test for genome-wide association studies. Am J Hum Genet. 2010; 87:139-145. [PubMed: 20598278]

Lux AL, Edwards SW, Hancock E, Johnson AL, Kennedy CR, Newton RW, O’Callaghan FJ, Verity CM, Osborne JP. The United Kingdom Infantile Spasms Study comparing vigabatrin with prednisolone or tetracosactide at 14 days: a multicentre, randomised controlled trial. Lancet. 2004; 364:1773-1778. [PubMed: 15541450]

Manolio TA, Brooks LD, Collins FS. A HapMap harvest of insights into the genetics of common disease. J Clin Invest. 2008; 118:1590-1605. [PubMed: 18451988]

Martinez G, Wijesinghe M, Turner K, Abud HE, Taketo MM, Noda T, Robinson ML, de Iongh RU. Conditional mutations of beta-catenin and APC reveal roles for canonical Wnt signaling in lens differentiation. Invest Ophthalmol Vis Sci. 2009; 50:4794-4806. [PubMed: 19515997]

Maurer GD, Brucker DP, Bähr O, Harter PN, Hattingen E, Walenta S, Mueller-Klieser W, Steinbach JP, Rieger J. Differential utilization of ketone bodies by neurons and glioma cell lines: a rationale for ketogenic diet as experimental glioma therapy. BMC Cancer. 2011; 11:315. [PubMed: 21791085]

Maurus D, Héligon C, Bürger-Schwärzler A, Brändli AW, Kühl M. Noncanonical Wnt-4 signaling and EAF2 are required for eye development in Xenopus laevis. EMBO J. 2005; 24:1181-1191. [PubMed: 15775981]

Moreno MC, de Zavalía N, Sande P, Jaliffa CO, Fernandez DC, Keller Sarmiento MI, Rosenstein RE. Effect of ocular hypertension on retinal GABAergic activity. Neurochem Int. 2008; 52:675-682. [PubMed: 17928106]

Nguyen D, Alavi MV, Kim KY, Kang T, Scott RT, Noh YH, Lindsey JD, Wissinger B, Ellisman MH, Weinreb RN, Perkins GA, Ju WK. A new vicious cycle involving glutamate excitotoxicity, oxidative stress and mitochondrial dynamics. Cell Death Dis. 2011; 2:e240. [PubMed: 22158479]

Okumichi H, Mizukami M, Kiuchi Y, Kanamoto T. GABA A receptors are associated with retinal ganglion cell death induced by oxidative stress. Exp Eye Res. 2008; 86:727-733. [PubMed: 18336815]

Purcell S, Neale B, Todd-Brown K, Thomas L, Ferreira MA, Bender D, Maller J, Sklar P, de Bakker PI, Daly MJ, Sham PC. PLINK: a tool set for whole-genome association and population-based linkage analyses. Am J Hum Genet. 2007; 81:559-575. [PubMed: 17701901]

Quigley HA. Glaucoma. Lancet. 2011; 377:1367-1377. [PubMed: 21453963]

Quigley HA, Broman AT. The number of people with glaucoma worldwide in 2010 and 2020. Br J Ophthalmol. 2006; 90:262-267. [PubMed: 16488940]

Racette L, Wilson MR, Zangwill LM, Weinreb RN, Sample PA. Primary open-angle glaucoma in blacks: a review. Surv Ophthalmol. 2003; 48:295-313. [PubMed: 12745004]

Ramdas WD, Wolfs RC, Kiefte-de Jong JC, Hofman A, de Jong PT, Vingerling JR, Jansonius NM. Nutrient intake and risk of open-angle glaucoma: the Rotterdam Study. Eur J Epidemiol. 2012; 27(5):385-393. [PubMed: 22461101] 
Sedel F, Challe G, Mayer JM, Boutron A, Fontaine B, Saudubray JM, Brivet M. Thiamine responsive pyruvate dehydrogenase deficiency in an adult with peripheral neuropathy and optic neuropathy. $\mathrm{J}$ Neurol Neurosurg Psychiatry. 2008; 79:846-847. [PubMed: 18559466]

Shin DH, Becker B, Kolker AE. Family history in primary open-angle glaucoma. Arch Ophthalmol. 1977; 95:598-600. [PubMed: 849183]

Sullivan TA, Geisert EE, Templeton JP, Rex TS. Dose-dependent treatment of optic nerve crush by exogenous systemic mutant erythropoietin. Exp Eye Res. 2012; 96:36-41. [PubMed: 22306016]

Suzuki M, Meguro A, Ota M, Nomura E, Kato T, Nomura N, Kashiwagi K, Mabuchi F, Iijima H, Kawase K, Yamamoto T, Nakamura M, Negi A, Sagara T, Nishida T, Inatani M, Tanihara H, Aihara M, Araie M, Fukuchi T, Abe H, Higashide T, Sugiyama K, Kanamoto T, Kiuchi Y, Iwase A, Ohno S, Inoko H, Mizuki N. Genotyping HLA-DRB1 and HLA-DQB1 alleles in Japanese patients with normal tension glaucoma. Mol Vis. 2010; 16:1874-1879. [PubMed: 21031025]

Thorleifsson G, Walters GB, Hewitt AW, Masson G, Helgason A, DeWan A, Sigurdsson A, Jonasdottir A, Gudjonsson SA, Magnusson KP, Stefansson H, Lam DS, Tam PO, Gudmundsdottir GJ, Southgate L, Burdon KP, Gottfredsdottir MS, Aldred MA, Mitchell P, St Clair D, Collier DA, Tang N, Sveinsson O, Macgregor S, Martin NG, Cree AJ, Gibson J, Macleod A, Jacob A, Ennis S, Young TL, Chan JC, Karwatowski WS, Hammond CJ, Thordarson K, Zhang M, Wadelius C, Lotery AJ, Trembath RC, Pang CP, Hoh J, Craig JE, Kong A, Mackey DA, Jonasson F, Thorsteinsdottir U, Stefansson K. Common variants near CAV 1 and CAV 2 are associated with primary open-angle glaucoma. Nat Genet. 2010; 42:906-909. [PubMed: 20835238]

van der Veen RL, Fuijkschot J, Willemsen MA, Cruysberg JR, Berendschot TT, Theelen T. Patients with Sjögren-Larsson syndrome lack macular pigment. Ophthalmol. 2010; 117:966-971.

Wan G, Mathur R, Hu X, Liu Y, Zhang X, Peng G, Lu X. Long non-coding RNA ANRIL (CDKN2BAS) is induced by the ATM-E2F1 signaling pathway. Cell Signal. 2013; 25:1086-1095. [PubMed: 23416462]

Wax MB. The case for autoimmunity in glaucoma. Exp Eye Res. 2011; 93:187-190. [PubMed: 20801114]

Wiggs JL, Kang JH, Yaspan BL, Mirel DB, Laurie C, Crenshaw A, Brodeur W, Gogarten S, Olson LM, Abdrabou W, DelBono E, Loomis S, Haines JL, Pasquale LR. GENEVA Consortium. Common variants near CAV 1 and CAV 2 are associated with primary open-angle glaucoma in Caucasians from the USA. Hum Mol Genet. 2011; 20:4707-4713. [PubMed: 21873608]

Wiggs JL, Yaspan BL, Hauser MA, Kang JH, Allingham RR, Olson LM, Abdrabou W, Fan BJ, Wang DY, Brodeur W, Budenz DL, Caprioli J, Crenshaw A, Crooks K, Delbono E, Doheny KF, Friedman DS, Gaasterland D, Gaasterland T, Laurie C, Lee RK, Lichter PR, Loomis S, Liu Y, Medeiros FA, McCarty C, Mirel D, Moroi SE, Musch DC, Realini A, Rozsa FW, Schuman JS, Scott K, Singh K, Stein JD, Trager EH, Vanveldhuisen P, Vollrath D, Wollstein G, Yoneyama S, Zhang K, Weinreb RN, Ernst J, Kellis M, Masuda T, Zack D, Richards JE, Pericak-Vance M, Pasquale LR, Haines JL. Common variants at 9p21 and 8q22 are associated with increased susceptibility to optic nerve degeneration in glaucoma. PLoS Genet. 2012; 8:e1002654. [PubMed: 22570617]

Wiggs JL, Hauser MA, Abdrabou W, Allingham RR, Budenz DL, Delbono E, Friedman DS, Kang JH, Gaasterland D, Gaasterland T, Lee RK, Lichter PR, Loomis S, Liu Y, McCarty C, Medeiros FA, Moroi SE, Olson LM, Realini A, Richards JE, Rozsa FW, Schuman JS, Singh K, Stein JD, Vollrath D, Weinreb RN, Wollstein G, Yaspan BL, Yoneyama S, Zack D, Zhang K, PericakVance M, Pasquale LR, Haines JL. The NEIGHBOR consortium primary open-angle glaucoma genome-wide association study: rationale, study design, and clinical variables. J Glaucoma. 2013; 22:517-525. [PubMed: 22828004]

Willer CJ, Li Y, Abecasis GR. METAL : fast and efficient meta-analysis of genomewide association scans. Bioinformatics. 2010; 26:2190-2191. [PubMed: 20616382]

Wyatt AW, Osborne RJ, Stewart H, Ragge NK. Bone morphogenetic protein 7 (BMP7) mutations are associated with variable ocular, brain, ear, palate, and skeletal anomalies. Hum Mutat. 2010; 31:781-787. [PubMed: 20506283]

Yaspan BL, Bush WS, Torstenson ES, Ma D, Pericak-Vance MA, Ritchie MD, Sutcliffe JS, Haines JL. Genetic analysis of biological pathway data through genomic randomization. Hum Genet. 2011; 129:563-571. [PubMed: 21279722] 
Yu DY, Cringle SJ, Balaratnasingam C, Morgan WH, Yu PK, Su EN. Retinal ganglion cells: energetics, compartmentation, axonal transport, cytoskeletons and vulnerability. Prog Retin Eye Res. 2013; 36:217-246. [PubMed: 23891817]

Zhang C, McCall MA. Receptor targets of amacrine cells. Vis Neurosci. 2012; 29:11-29. [PubMed: 22310370] 


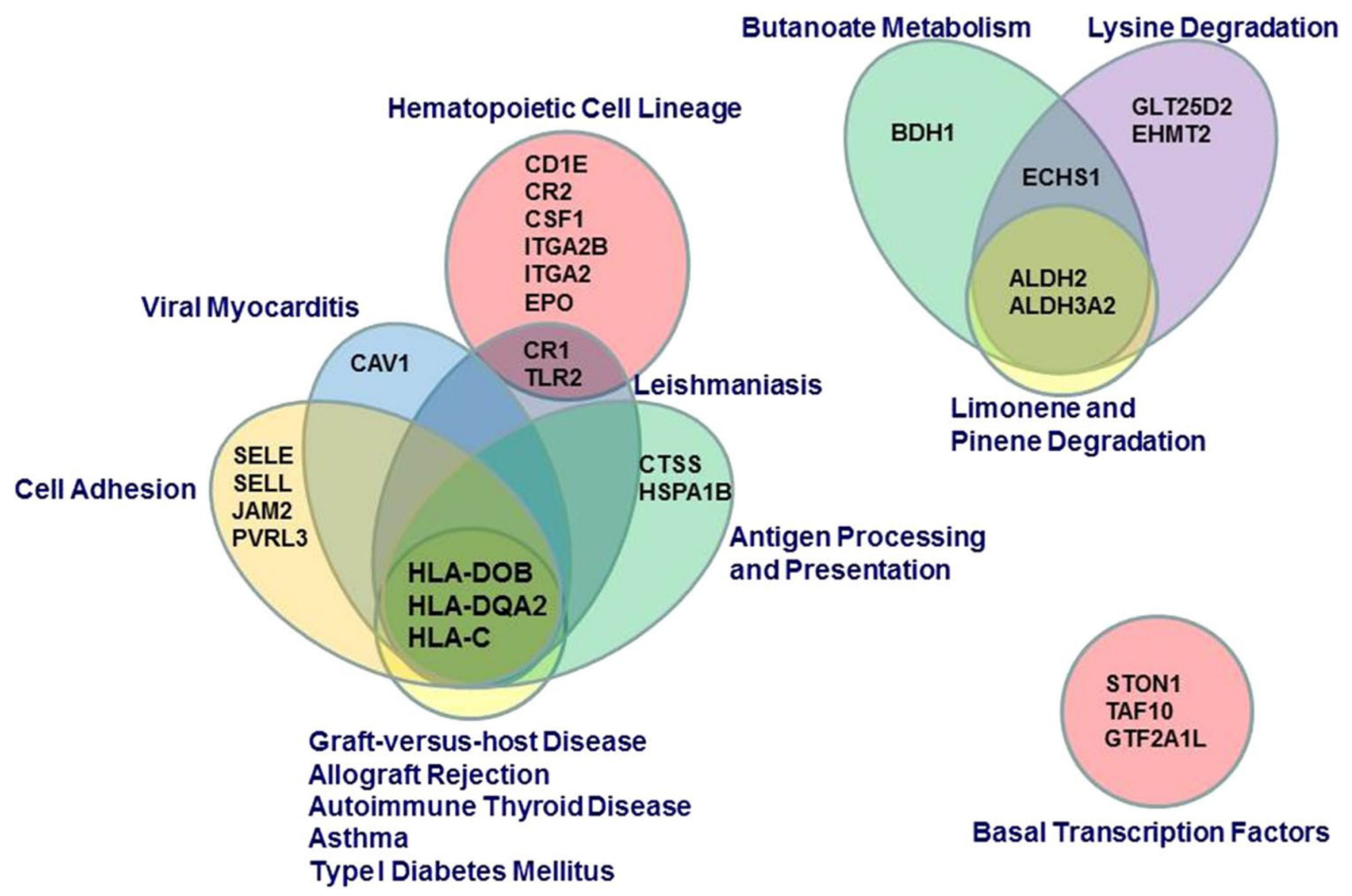

Fig. 1.

KEGG pathways significantly associated with POAG overall. Pathways with a permutated $p$ of $<0.001$ are presented (blue font). The individual genes within the associated pathways with gene-based permutated $p$ values $<0.001$ are listed in black font and are contained in the ellipse corresponding to the pathway. Genes that are present in more than one pathway are indicated by overlapping ellipses. Nine pathways contained the 3 associated HLA genes $H L A-D O B, H L A-D Q A 2$ and $H L A-C . A L D H 2$ and $A L D H 3 A 2$ were found in three pathways: (butanoate metabolism, lysine degradation, and limonene and pinene degradation). Two genes in the Leishmaniasis pathway overlap with the hematopoietic cell lineage pathway. The significantly associated genes in the basal transcription factor pathway were independent of all other pathways. POAG Primary open-angle glaucoma; SELE selectin E; SELL selectin L; JAM2 junctional adhesion molecule 2; $P V R L 3$ poliovirus receptor-related 3; $C A V 1$ caveolin 1; HLA-DOB, HLA-DQA2, HLA-C human leukocyte antigen- DOB, DQA2, C; $C D E 1$ T cell surface glycoprotein CD1e; $C R 2$ complement receptor 2; CR1 complement receptor 1; CSF1 colony-stimulating factor 1; ITGA2B integrin alpha chain $2 \mathrm{~b}$; ITGA2 integrin alpha chain 2; EPO erythropoietin; TLR2 toll-like receptor 2; CTSS cathepsin S; HSPA1B heat shock $70 \mathrm{kDa}$ protein 1B; BDHI 3-hydroxybutyrate dehydrogenase, type 1; ECHS1 enoyl-Coenzyme A hydratase, short chain, 1; ALDH2 aldehyde dehydrogenase 2; ALDH3A2 aldehyde dehydrogenase 3A2; GLT25D2 glycosyltransferase 25 domain containing 2; EHMT2 euchromatic histone-lysine $\mathrm{N}$ - 
methyltransferase 2; STON1 stonin 1; TAF10 TATA box binding protein (TBP)-associated factor; GTF2A1L general transcription factor IIA, 1-like 

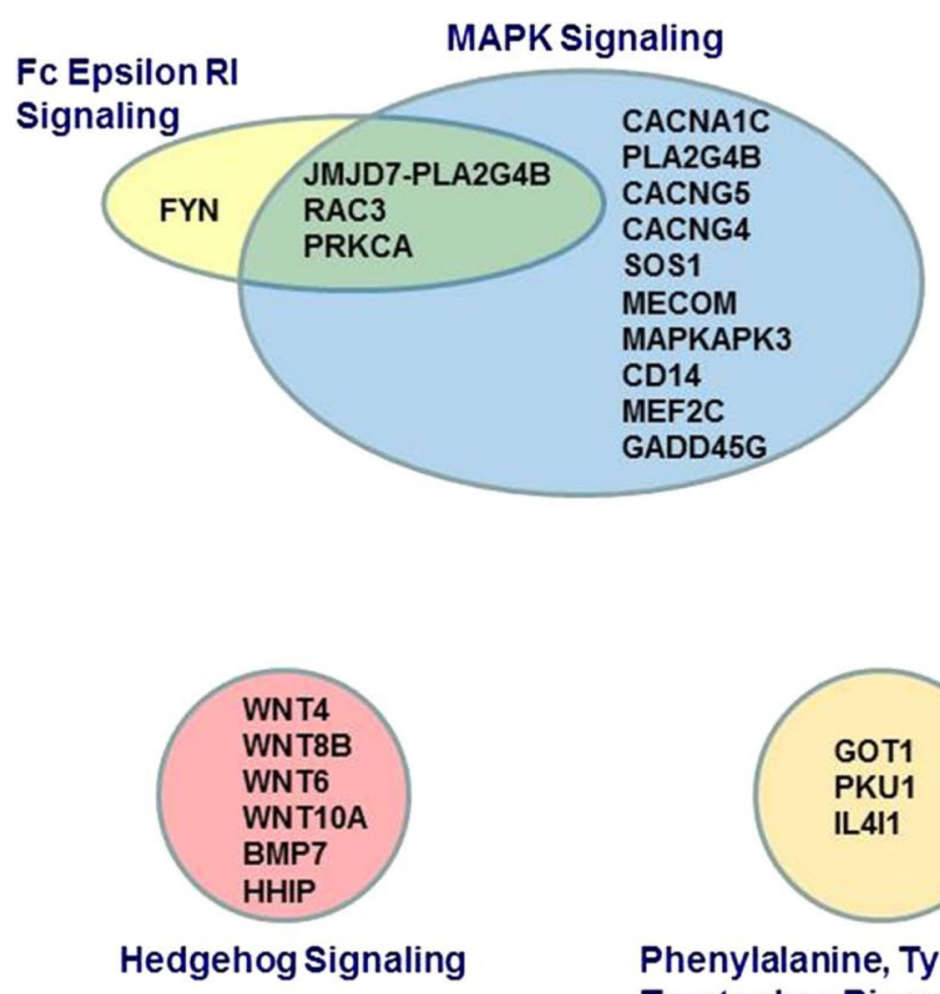

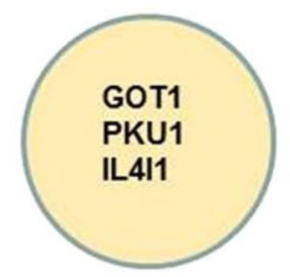

Phenylalanine, Tyrosine and Tryptophan Biosynthesis

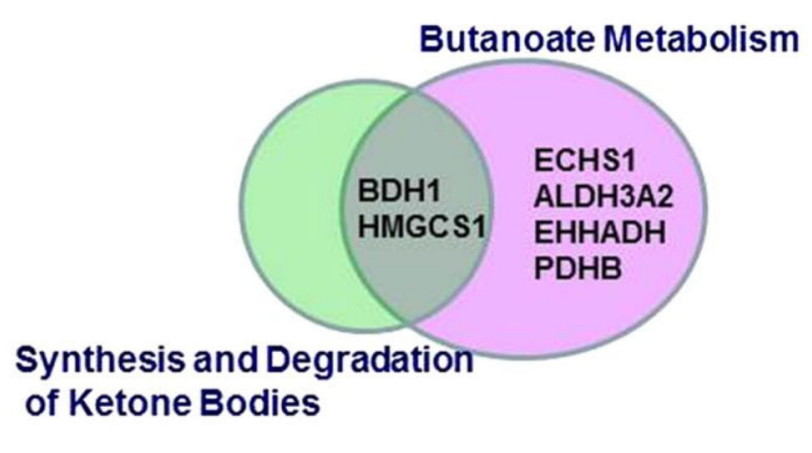

Fig. 2.

KEGG pathways significantly associated with NPG. Pathways with a permutated $p$ of $<0.001$ are presented (blue font). The individual genes within the associated pathways with gene-based permutated $p$ values $<0.001$ are listed in black font and are contained in the ellipse corresponding to the pathway. Genes that are present in more than one pathway are indicated by overlapping ellipses. With the exception of $F Y N$, the genes with significant association in the Fc epsilon RI signaling pathway are also included in the MAPK signaling pathway. Similarly, both of the significant genes in the synthesis and degradation of ketone bodies pathway (BDH1, HMGCS1) are also found in the butanoate pathway. The significantly associated genes in the Hedgehog signaling pathway, the phenylalanine, tyrosine and tryptophan biosynthesis pathway and the glycosaminoglycan biosynthesisheparan sulfate pathway did not overlap with any other pathway. $N P G$ normal-pressure glaucoma; FYN tyrosine-protein kinase Fyn; JMJD7-PLA2G4B jumonji domain containing 7-phospholipase $\mathrm{A} 2$, group IVB (cytosolic) read-through; $R A C 3$ ras-related $\mathrm{C} 3$ botulinum toxin substrate 3; PRKCA protein kinase $\mathrm{C}$, alpha; CACNAIC calcium channel, voltagedependent, L type, alpha 1C subunit; PLA2G4B phospholipase A2, group IVB (cytosolic); CACNG5 calcium channel, voltage-dependent, gamma subunit 5; CACNG4 calcium channel, voltage-dependent, gamma subunit 4; SOS1 son of sevenless homolog 1; MECOM MDS1 and EVI1 complex locus; MAPKAPK3 mitogen-activated protein kinase-activated protein kinase 3; $C D 14$ cluster of differentiation 14; $M E F 2 C$ myocyte enhancer factor 2C; $G A D D 45 G$ growth arrest and DNA -damage-inducible, gamma; $B D H 1$ 3-hydroxybutyrate dehydrogenase, type 1; HMGCS1 3-hydroxy-3-methylglutaryl-Coenzyme A synthase 1; ECHS1 enoyl-Coenzyme A hydratase, short chain, 1; ALDH3A2 aldehyde dehydrogenase 
3A2; EHHADH enoyl-Coenzyme A, hydratase/3-hydroxyacyl-Coenzyme A dehydrogenase; $P D H B$ pyruvate dehydrogenase (lipoamide) beta; WNT4 wingless-type MMTV integration site family, member 4; $W N T 8 B$ wingless-type MMTV integration site family, member $8 \mathrm{~b}$; WNT6 wingless-type MMTV integration site family, member 6; WNT10A wingless-type MMTV integration site family, member 10a; BMP7 bone morphogenetic protein 7; HHIP hedgehog interacting protein; GOT1 glutamic-oxaloacetic transaminase 1; PKU1 phenylalanine hydroxylase; ILAII interleukin 4 induced 1; EXT2 exostosin glycosyltransferase 2; XYLT1 xylosyltransferase I 


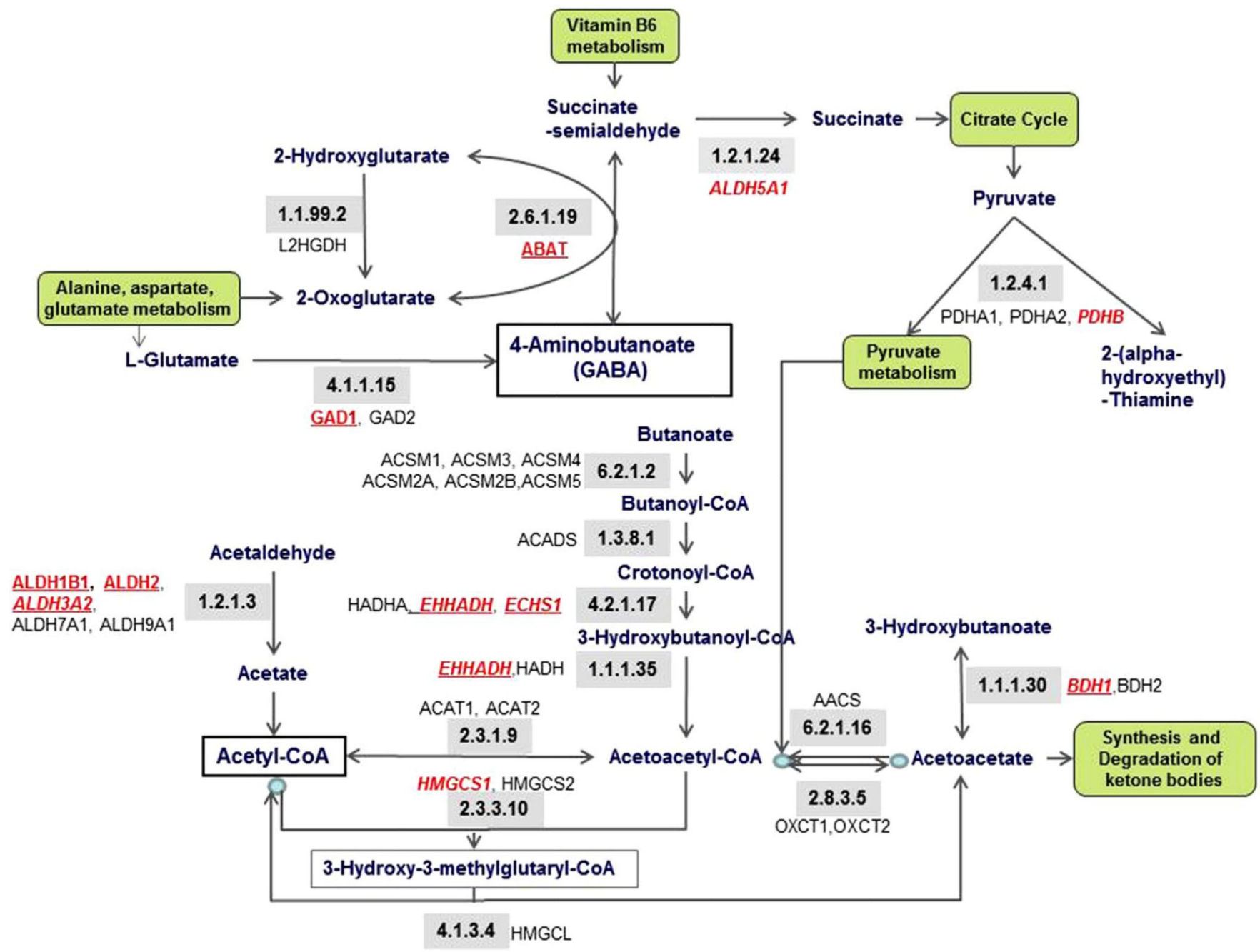

Fig. 3.

Butanonate metabolic pathway (KEGG pathway hsa00650). The biochemical pathways involved in butanonate metabolism are depicted. Individual genes with at least nominal association $(p<0.05)$ with POAG are indicated by underlined red font and those with at least nominal association with NPG by italic red font. Genes associated with both POAG and NPG are underlined and italic red font. Actual $p$ values are presented in Table 3. $A K R 1 B 10$ (aldo-keto reductase1, B10) is part of an auxiliary pathway that impacts acetylCoA metabolism that is not shown in this figure 


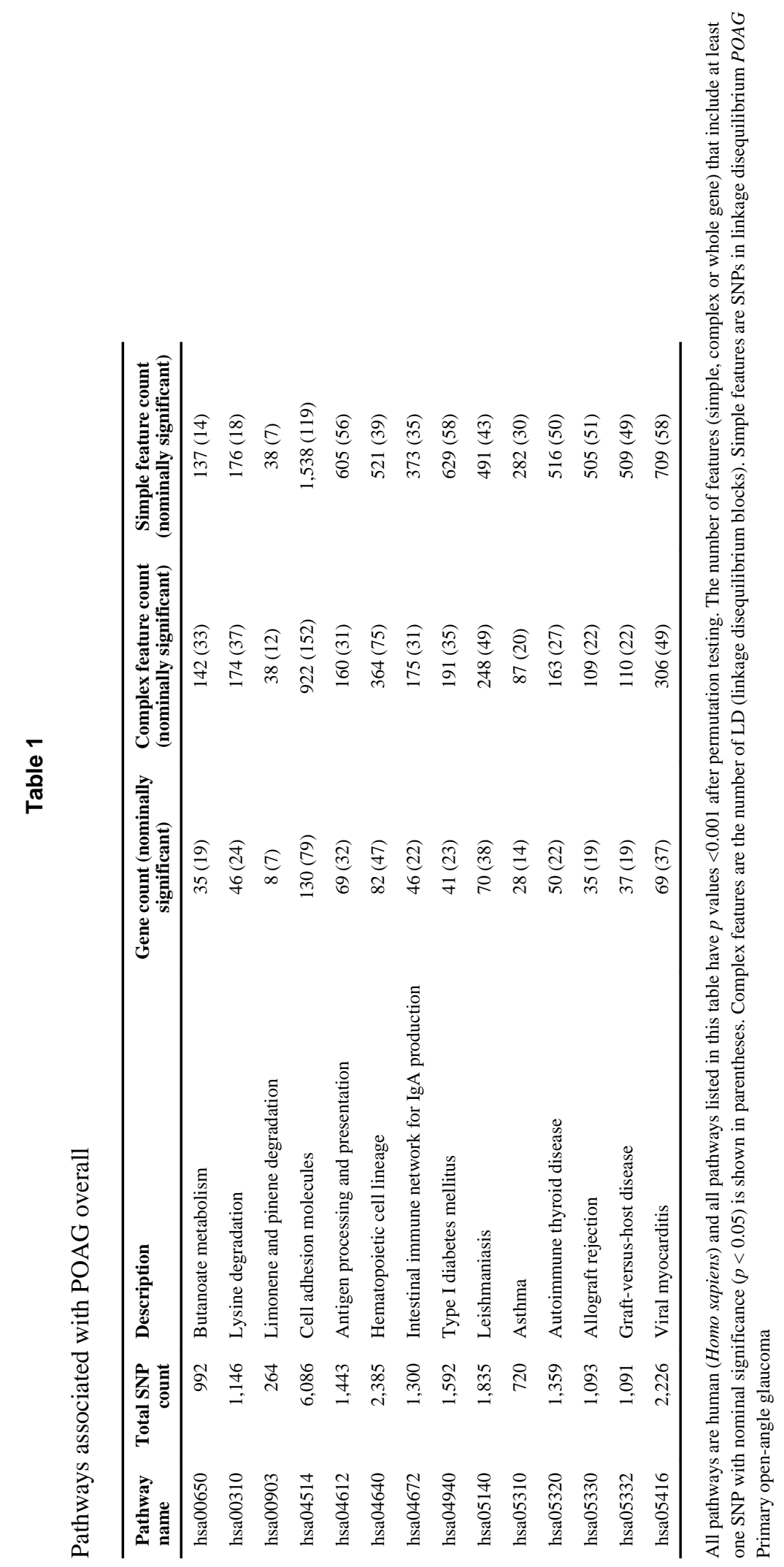




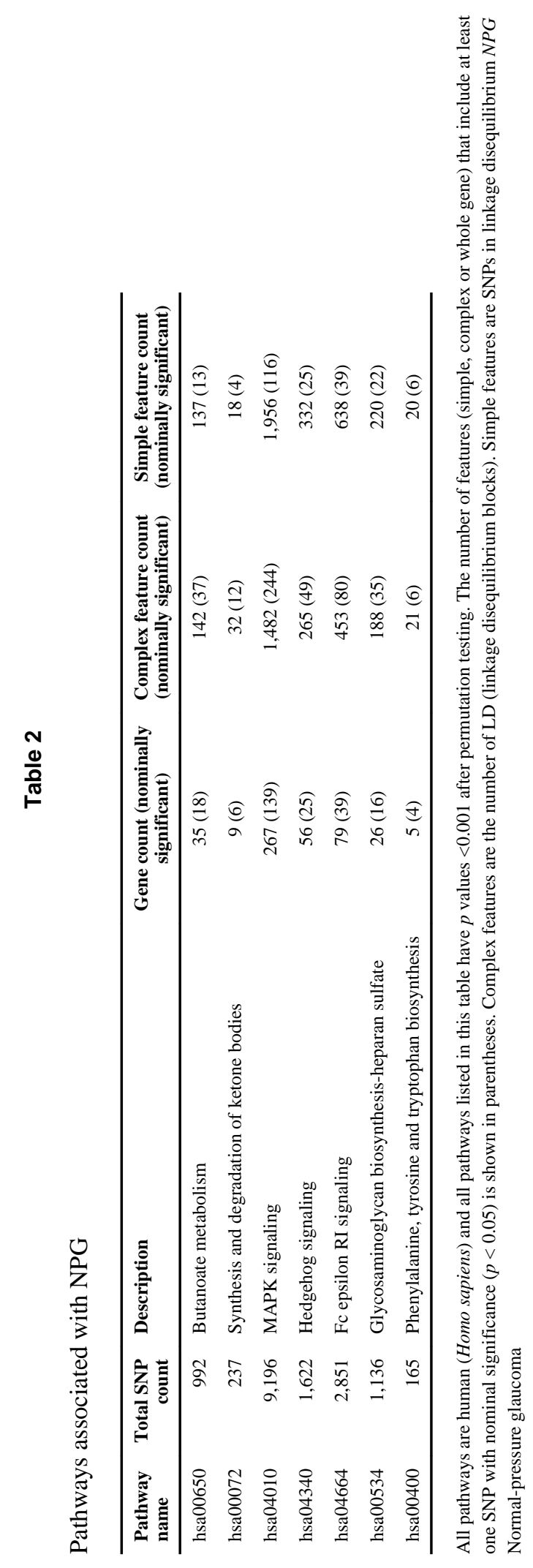

Hum Genet. Author manuscript; available in PMC 2015 October 01. 


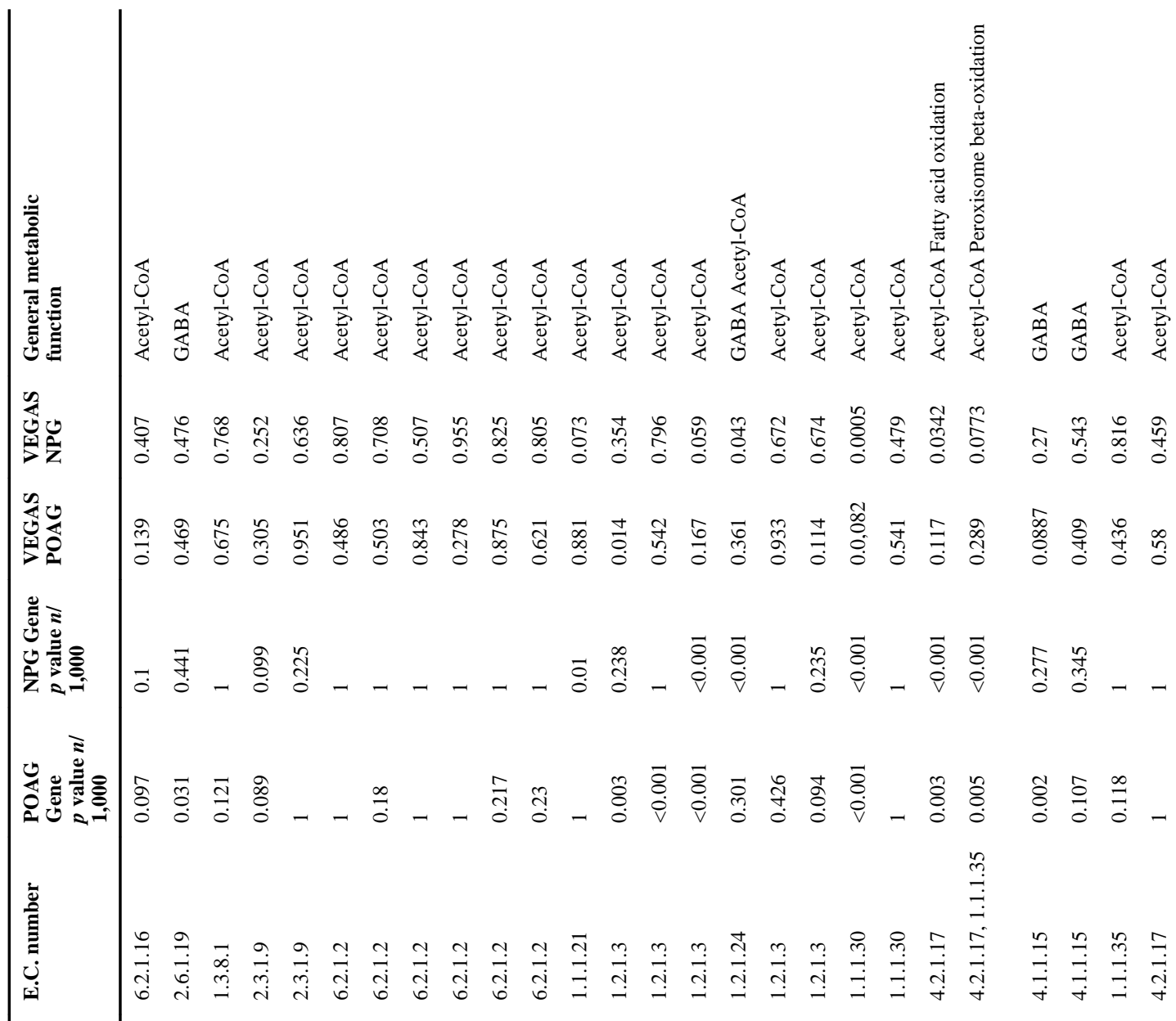




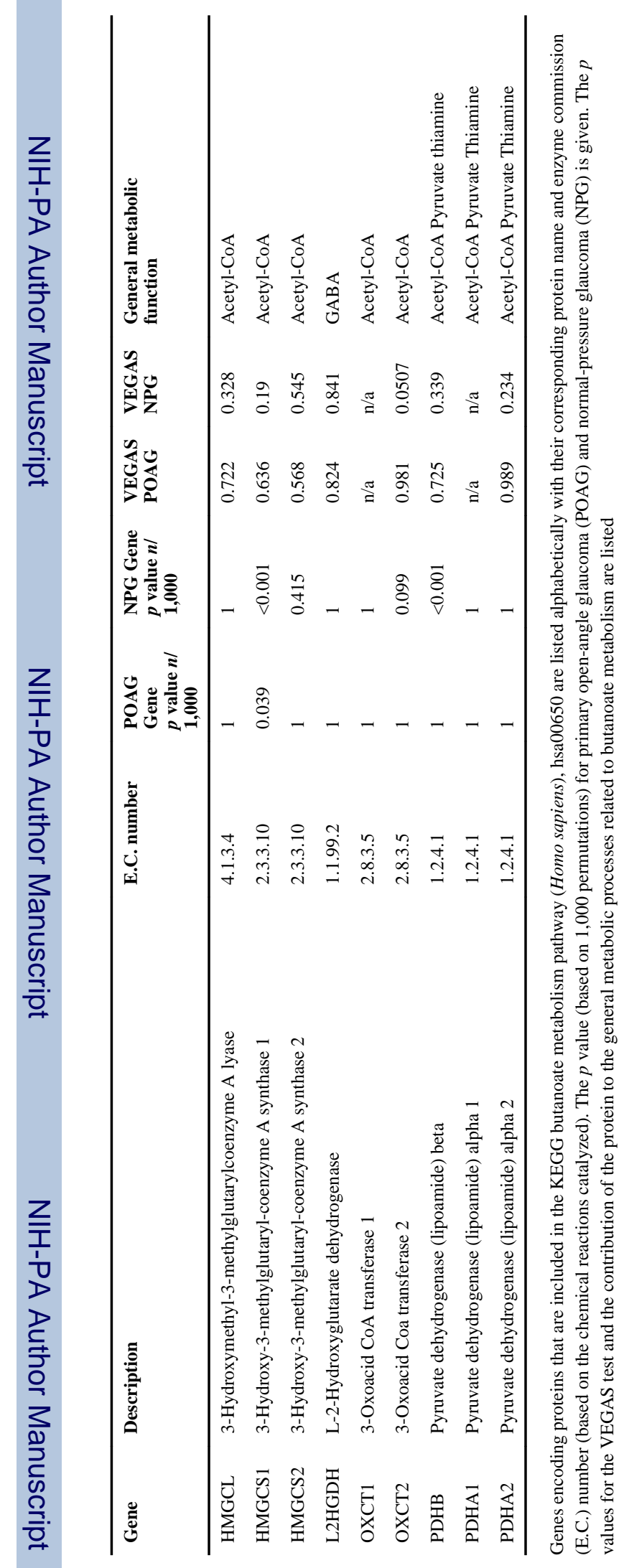

Hum Genet. Author manuscript; available in PMC 2015 October 01. 